
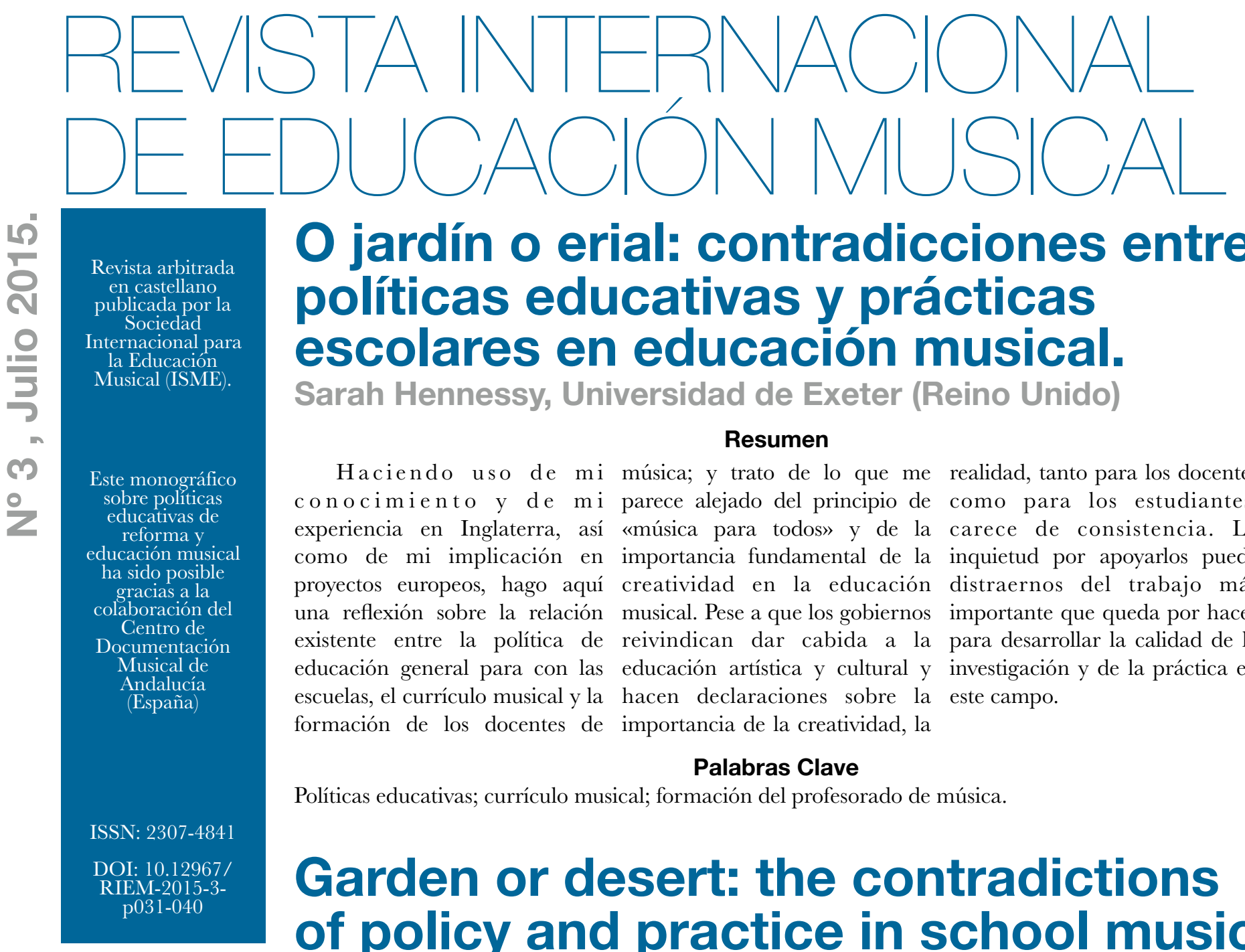

\title{
O jardín o erial: contradicciones entre políticas educativas y prácticas escolares en educación musical.
} Sarah Hennessy, Universidad de Exeter (Reino Unido)

\section{Resumen}

Haciendo uso de mi música; y trato de lo que me realidad, tanto para los docentes conocimiento y de mi parece alejado del principio de como para los estudiantes, experiencia en Inglaterra, así «música para todos» y de la carece de consistencia. La como de mi implicación en importancia fundamental de la inquietud por apoyarlos puede proyectos europeos, hago aquí creatividad en la educación distraernos del trabajo más una reflexión sobre la relación musical. Pese a que los gobiernos importante que queda por hacer existente entre la política de reivindican dar cabida a la para desarrollar la calidad de la educación general para con las educación artística y cultural y investigación y de la práctica en escuelas, el currículo musical y la hacen declaraciones sobre la este campo.

formación de los docentes de importancia de la creatividad, la

\section{Palabras Clave}

Políticas educativas; currículo musical; formación del profesorado de música.

\section{Garden or desert: the contradictions of policy and practice in school music education.}

\section{Sarah Hennessy, Exeter University (United Kingdom)}

\section{Abstract}

Using my knowledge and discuss what I see as a retreat of creativity, the reality for experience in England and my from the principle of 'music for teachers and students is involvement in European all' and from the central inconsistent. The preoccupation project work, I reflect upon the importance of creativity in music with advocacy can distract us relationship between general education. Whilst governments from the more important work education policy towards claim to embrace arts and to be done in developing the schools, the music curriculum, cultural education; and make quality of research and practice and music teacher training; and statements about the importance in the field.

\section{Keywords}

Policy; music curriculum; music teacher training.

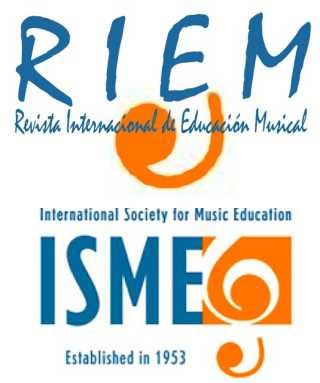


 políticas educativas y prácticas escolares en educación musical.}

\author{
por Sarah Hennessey, Universidad de Exeter (Reino Unido).
}

Los formadores de música tienden a actuar con un cierto grado de aislamiento unos de otros, bien sea en la escuela, en el seno de la comunidad, en las enseñanzas universitarias o en las clases particulares. Este aislamiento puede conducir a una falta de conocimiento y de sentido crítico de la práctica de uno mismo -y de los demás-, aunque también puede apartarnos de la línea central del debate y de la política educativa. Desde una perspectiva norteamericana, Paul Woodford (2005) comentó que:

Desgraciadamente hace mucho tiempo que los docentes de música abandonaron o fueron abandonados por la esfera pública y se refugiaron en el relativo aislamiento de su profesión mientras perdían el contacto con los ideales y con los movimientos políticos más amplios que tiempos atrás habían sido su fuente de inspiración (p. 57) .

Continuaba comentando que los docentes de música no han desarrollado las ideas que habían sido objeto de tan intensos debates en los años 60 y 70 en relación con la educación musical para todos y que se han refugiado en los modelos de currículo y de enseñanza basados en la práctica tradicional.

No quiero sugerir que este comentario pueda aplicarse a todos los docentes de música ni en todas partes - me refiero a los principios de la música para todos, las pedagogías inclusivas y las democráticas-; la creatividad y la diversidad cultural pueden encontrarse vivas en muchos otros países -en el trabajo de formadores de docentes y el de destacados profesionales- pero, desde una perspectiva inglesa, veo una tendencia preocupante, promovida y apoyada por los diseñadores de políticas, orientada hacia un enfoque más limitado y conservador de la educación musical en las escuelas. También veo un número de trabajadores cada vez más dóciles, sin voluntad, o bien carentes de las competencias necesarias para desafiar estas tendencias. El aislamiento experimentado por muchos de ellos sirve para reducir la posibilidad de alzar una voz crítica colectiva que, antes que la obediencia, busca generar propuestas significativas, inclusivas e innovadoras para la educación musical.

Todos estamos sujetos a unos imperativos en nuestras condiciones de trabajo, a los contextos musicales y educativos para nuestro trabajo, a los recursos disponibles, y a una relativa organización y autonomía que nos permitan trabajar de forma que se reflejen nuestros conocimientos y valores profesionales. Si queremos no solo sostener y desarrollar este campo, sino también influenciar y desafiar a las políticas que afectan directamente a la naturaleza y a la calidad de la educación musical a la que aspiramos es esencial desarrollar una perspectiva clara y crítica sobre lo que valoramos y sobre lo que hacemos.

Woodford evoca los años 60 y 70, una época (por lo menos en Norteamérica) en la que la educación se fue modelando conforme a las nuevas ideas liberales y democráticas. No estoy segura de que ninguno de nosotros pueda afirmar que el clima político actual busca los mismos ideales para la educación del siglo XXI. Existe esa retórica, sin duda (especialmente en torno a la creatividad y a la voz del alumnado), pero el contexto ha cambiado mucho. Según Carter y O'Neill (1995, citado en Ball, 1998), se puede identificar un cambio en la relación entre políticas, gobierno y educación en los países occidentalizados y postindustrializados. Resaltan cinco elementos o imperativos principales que orientan este cambio:

1. Mejorar la economía nacional reforzando los vínculos entre la enseñanza, el empleo, la productividad y el comercio.

2. Aumentar los resultados de los estudiantes en cuanto a sus destrezas y competencias en relación con el empleo.

3. Lograr tener más control directo sobre el contenido del plan de estudios y de la evaluación.

4. Reducir los costes en los ministerios de educación.

5. Aumentar la aportación de la comunidad a la educación mediante una implicación más directa en la toma de decisiones en el ámbito escolar, así como en las presiones de la oferta de mercado. (p. 122)

Reconozco claramente estos cinco elementos en el contexto actual de Inglaterra y veo también de qué manera tienen un impacto en la educación musical de forma directa e indirecta. En los últimos 20 años hemos experimentado una creciente centralización en todos los aspectos de la legislación de la enseñanza y de las enseñanzas universitarias. Las contradicciones y tensiones generadas en un afán por lograr un mayor control así como por abrirse, al mismo tiempo, a un mercado en el que escuelas (y universidades) compitan activamente por captar estudiantes, se hacen claramente evidentes. La utilización de un modelo de negocio para la educación obliga a tener una mentalidad de ganadores y perdedores creando así unas diferencias espectaculares en la manera en la que las diferentes instituciones responden. Muchas de estas instituciones prosperan, pero también son muchas las que luchan; algunas de ellas se encuentran inmersas en graves dificultades, amenazadas por el cierre. En este contexto, en la escuela, la música y las artes pueden llegar a ser una verdadera fuente de grandes expectativas y logros, de innovación y diseño curricular imaginativo mientras, en otro contexto, pueden naufragar sin apenas dejar rastro alguno. Con la introducción del Currículo Nacional inglés en 1990 (1992 para la música) se quería asegurar la consistencia de la capacitación y elevar el nivel en todas las escuelas. Las sucesivas políticas (demasiadas para hacerlas constar todas aquí) guiadas por los imperativos a los que nos hemos referido anteriormente hicieron que esto fuera inalcanzable en una asignatura como la música.

La globalización también tiene aquí un papel significativo. Mientras existen unos esfuerzos que han sido concertados con el fin de lograr una Educación Primaria «básica» para todos, existe también el imperativo (en el mundo desarrollado) de competir mediante la competitividad internacional y la utilización de la educación para permitir que una economía nacional aventaje a otra (Chawla-Duggan y Lowe, 2008). En algunos países el «mercado» y el individualismo impulsan a la educación privada (especialmente en el Reino Unido), contribuyendo a acentuar 
una brecha ya importante entre ricos y pobres ${ }^{1}$. Según muchos educadores, tal competitividad tiene un efecto dañino:

[Tiene un efecto dañino] El trabajo de algunos analistas de política «global», alejados de las complejidades existentes en las aulas y de las conclusiones desconcertantes de los investigadores que representan un alto grado de dificultad para los políticos en busca de «transformaciones» rápidas del sistema escolar antes de las próximas elecciones. Adoptan [...] un modelo de [...] caracterizado por una implacable presión, competitividad, supervisión, servicio al cliente, datos para la gestión del rendimiento, la responsabilidad y la relación calidad/precio; y autonomía profesional para los docentes únicamente cuando haya sido otorgada por el centro [...]. Su noción de enseñanza está concebida con estrechez de miras y tecnocracia [...]. $\mathrm{Su}$ modelo sigue siendo poco sofisticado, impracticable y no es democrático [...]. Sus recomendaciones son educativa y socialmente disfuncionales y no deberían ser parte de una reforma escolar en una democracia (Coffield, 2012, p. 145).

El efecto PISA o, más bien, el «golpe PISA», que proviene de países que consiguen resultados mucho menos satisfactorios en las comparaciones internacionales de lo que esos mismos países esperan (Baird y otros, 2011), juega un papel significativo en este contexto; aunque las Artes no formen parte de las pruebas que se centran en el rendimiento en lectura, matemáticas y ciencias en estudiantes de 15 años, pueden tener consecuencias directas. Alexander (2012) argumenta que el problema no es PISA en sí, sino cómo los diseñadores de políticas interpretan y generan políticas a partir de estos datos. Y se pregunta: "los resultados de PISA ¿en cuántos países han sido explotados o manipulados con fines políticos?" (p. 8). Al mismo tiempo, Baird y otros (2011, p. 2) observan que "es llamativo que los resultados de PISA parezcan haber sido utilizados para elevar una retórica política patriotera con el fin de llevar a cabo reformas educativas en algunos países".

Las declaraciones realizadas por el Ministerio de Educación en Inglaterra lo ilustran rigurosamente: El "informe PISA subraya la urgente necesidad de reformar nuestro sistema escolar. Necesitamos aprender de los países que logran alcanzar el más alto rendimiento. Tan solo mediante una reforma del sistema en su conjunto podremos transformar la educación para hacer de nuestra nación uno de los países de máximo rendimiento"2.

El actual Ministerio de Educación ha utilizado el escurridizo puesto de Inglaterra en las clasificaciones en matemáticas y ciencias para introducir nuevas políticas que afectan a la formación del profesorado, limitan el plan de estudios y modifican la configuración de los exámenes. En un clima marcado por las leyes del mercado y de recesión económica es difícil organizar una debate contra tales gestiones tecnocráticas y, según parece, nadie -excepto la comunidad educativa- cuestiona que tenemos que tratar de alcanzar «lo más alto» a toda costa, y con la atención puesta exclusivamente en los planteamientos profesionales que se adecúan a este imperativo.

Una consecuencia alarmante ha sido la introducción del Bachillerato inglés (EBACG o English Baccalaureat) en 2015. Se trata, para los institutos, de una nueva medición de los rendimientos del alumnado de 16 años en inglés, matemáticas, ciencias, historia o geografía y una lengua moderna. Con la exclusión de las artes, la introducción de estas políticas está teniendo ya un impacto sobre la contratación de profesorado de artes en los institutos de Enseñanza Secundaria y en una reducción de horario en todas las asignaturas relativas a las materias artísticas en el plan de estudios (Greevy y otros, 2012). Unos informes de prensa sugieren que algunas escuelas están reduciendo el perfil de la música, especialmente con respecto a las opciones que se ofrecen a los estudiantes más talentosos, y que se ha producido un descenso general en el número de matriculados en cursos de arte $^{3}$. Se ha organizado una campaña activa para incluir las artes en el Bachillerato inglés (EBACG), pero aún no se aprecia señal alguna de cambio de dirección por parte del gobierno.

Evidentemente, no estamos solos en esta «carrera hacia la cima». A modo de ejemplo, el «golpe» PISA en Alemania ha llevado a emprender importantes reformas, cuya consecuencia conduce a una reducción del currículo de Primaria para concentrarse en el lenguaje, las matemáticas y las ciencias. Esto viene acompañado por una incorporación de otras materias de modo que, en muchas partes de Alemania, la música ya no se enseña como asignatura independiente ${ }^{4}$.

\section{El impacto de las políticas en la educación musical}

Seguidamente quisiera tratar más de cerca cómo las políticas repercuten directa o indirectamente en la educación musical. He querido fijarme más en los problemas que en los éxitos sin negar que existen algunos aspectos muy buenos en cuanto a la educación musical en Inglaterra ${ }^{5}$, aunque sostengo que estos aspectos se dan frecuentemente pese a las decisiones políticas, más que gracias a ellas, y son también el resultado del compromiso y de la energía individuales y no tanto de una visión coherente y de una responsabilidad colectiva. Los ejemplos que presento ilustran cómo, por diseño o por defecto, las políticas tienen un impacto en la educación musical en las escuelas. Por preferencia propia me centraré más en la Educación Primaria aunque haga algunas referencias a la Secundaria. Al margen de mi interés personal, existe una buena razón para observar lo que ocurre en Primaria dado que, para la mayoría de los países, representa la etapa educativa en la que la música es accesible a todo el mundo. Su papel de socialización para los niños, de enculturación, así como de apoyo al desarrollo cognitivo general y físico (especialmente del lenguaje y la coordinación) está ampliamente reconocido en los documentos curriculares de los 20 países europeos incluidos en el proyecto $\operatorname{meNet}^{6} \mathrm{y}$, también, en los países anglófonos del mundo entero. Estos objetivos y aspiraciones se ven reflejados en la Hoja de Ruta para la Educación Artística, fruto de un congreso sobre Educación Artística: Construir capacidades creativas para el siglo XXI que tuvo lugar en Lisboa en 2006 y fue publicado por la UNESCO: "La educación artística contribuye en el desarrollo de una educación que integra las facultades físicas, intelectuales y creativas y que hace posible el desarrollo de relaciones más dinámicas y fructíferas entre la educación, la cultura y las artes" (p. 5).

Cada país en Europa (véase EACEA, 2009) incluye la música como asignatura reglada para todos los niños y niñas en la escuela Primaria y -hasta cierto punto- comparten unos objetivos curriculares similares (Shuayb y O'Donnell, 2008):

- Destrezas artísticas, conocimiento y comprensión. 
- Patrimonio cultural (identidad nacional).

- Pensamiento crítico (juicio estético).

- Expresión personal/identidad/desarrollo.

- Diversidad cultural (identidad europea/ concientización mundial).

- Creatividad (imaginación, resolución de problemas, asunción de riesgos).

A la hora de considerar la música por sí misma en la mayoría de los 20 países en los que nos fijamos dentro del proyecto meNet, la creatividad y la diversidad cultural están, sin duda, parcamente representadas: muy pocos currículos hacen referencia a la composición o a la improvisación por derecho propio. Predomina la adquisición de destrezas técnicas artísticas así como el conocimiento y la valorización del patrimonio cultural. También hemos observado que, en muchos países europeos existen, fundamentalmente, dos caminos a seguir: escuela de música (para todos) y escuelas de música especializadas que funcionan después de la escuela y los fines de semana (como alternativa adicional que los padres pueden tener costeándola). Esto puede reducir la «voz» de la educación musical dentro del horario escolar, ya sea porque considera al profesorado que la imparte menos especialista y, por lo tanto, con un estatus más bajo que los profesores de escuela de música, ya sea que, en las mentes de los padres o en la de los diseñadores de políticas educativas, la educación musical puede considerarse bien servida con esta oferta adicional especializada. Esto también alimenta la idea de que la educación musical está destinada únicamente al alumnado con talento.

Cualesquiera que sean los objetivos para la educación musical, la pregunta clave es, sin duda, ¿cómo se logran? La brecha que existe entre la visión, la aspiración y la retórica de los documentos normativos y su aplicación a la práctica profesional es, a menudo, inaceptablemente amplia, y se ha producido por las exigencias contradictorias que tienen las escuelas y algunos docentes, ya sea por la falta de una formación apropiada para el profesorado o por la existencia de unos recursos inadecuados ${ }^{7}$.

Una preocupación esencial debería ser que todos los niños tuvieran acceso a una enseñanza musical prolongada y de calidad en la escuela. Sin duda, lo que constituye la buena calidad requiere ser definido y me atrevería a afirmar que existe un amplio consenso bien establecido entre los formadores de música en Inglaterra sobre lo que constituye la buena calidad (y que no creo necesario tener que detallar aquí). De hecho, el Currículo Nacional de Música para Inglaterra (1992, y revisado en 1995 y 2000), pese a su concisión, ha captado bastante bien la variedad y la diversidad de las actividades y músicas con las que los formadores de música esperan trabajar. Las influencias de Paynter (1970) y de Swanwick (1988) tienen resonancia en este documento, así como en los recursos y esquemas de trabajo desarrollados por las editoriales y otros órganos que apoyan esta puesta en práctica. De este modo, hacer música creativa (que incluye la composición, la improvisación y la adaptación) forma el núcleo central del currículo para el alumnado de entre los 5 y los 14 años y los cursos de preparación de exámenes cuando se trata de una asignatura optativa, y después de los 14 años también atribuyen un valor equivalente tanto en la composición como en la ejecución y en la audición. El Currículo Nacional también espera que el contenido musical sea de gran envergadura desde el punto de vista de los estilos, géneros y tradiciones, y que el profesorado pueda darle a su alumnado oportunidades para aprender de diferentes maneras (individualmente, en pequeños grupos o en grandes).

La introducción del Currículo Nacional a principios de los años 90 formaba parte de un paquete de políticas más amplio que buscaba alcanzar una mayor consistencia en cuanto a capacitación y calidad en todo el país, y una mayor responsabilidad por parte de las escuelas y profesores. Esto condujo a una financiación por parte del gobierno y a la aportación de recursos para apoyar el desarrollo profesional de los trabajadores y -en lo que a la música se refiereespecialmente el de los maestros de enseñanza general en las escuelas Primarias a las que se les exigió (por ley) incorporar la música en el programa. Esto funcionó bien y durante unos pocos años se apreciaron sensibles mejoras en cuanto a la confianza de los maestros a la hora de enseñar música en el aula; incluso, la formación de nuevos maestros se centró enérgicamente en el desarrollo de la competencia y de la confianza. La preocupación por el modo de mejorar las aptitudes del profesorado de enseñanza general en la enseñanza de la música dio origen a varias publicaciones y estudios de investigación a lo largo de los años 90 y durante los años 2000 (véase, por ejemplo, Mills, 1992; Glover y Ward, 1998; Hennessy y otros, 2000; Holden y Button, 2006). Estos trabajos nos permiten alcanzar una buena comprensión de los problemas a los que se enfrenta el profesorado de enseñanza general y nos da unas recomendaciones sobre lo que se necesita. No obstante, disponemos de menos datos que nos indiquen de forma positiva en qué medida cualquier trabajo de formación resultante es eficaz a largo plazo (los costes de estudios longitudinales y la movilidad del profesorado dificultan la tarea). No obstante, los beneficios de principios de los años 90 fueron diluidos por el impacto de otras políticas y estrategias abordadas en el periodo siguiente. Dichas estrategias han tenido un impacto significativo no solo en cuanto a la prestación y la práctica, sino también en cuanto a las actitudes y las creencias sobre quién enseña y sobre lo que constituye el currículo de música.

He aquí dos ejemplos para ilustrarlo:

\section{Tiempo para la PPA}

La presión creciente sobre la cantidad de contenido del currículo de Primaria y sobre las exigencias para enfocar las asignaturas principales llevó a la decisión, en 2005, de dar a todos los maestros un tiempo fuera del aula reservado para la Planificación, la Preparación y la Evaluación (designado como PPA en inglés). Esto representa, aproximadamente, 2 horas por semana, y a las escuelas se les dio la libertad de idear sus propias soluciones en cuanto a quién se encargaría de la enseñanza en las aulas durante este tiempo. Muchas escuelas $^{8}$ dedicaron este periodo de tiempo al aprendizaje de la música, de modo que fuera otra persona diferente del maestro habitual quien enseñara en el aula. Esta persona puede variar, desde un profesor de música especialista cualificado o un profesor adjunto dotado de algo de confianza en el campo musical, hasta, por ejemplo, un pianista sin titulación ${ }^{9}$.

En Inglaterra, basamos aún la Educación Primaria en el principio según el cual el maestro de clase es responsable de la totalidad del programa; este es el principio a partir del que formamos a los docentes, y en el que se fundamenta el 
ก้ Currículo Nacional, pero la práctica en las escuelas es difusa actualmente y no está regulada. Desde luego, tal acuerdo podría ser muy satisfactorio, como en los casos de un profesor de música ofreciendo pericia y calidad constante a lo largo de la vida escolar, o un profesor bien integrado en la comunidad escolar haciendo posible una buena conexión con otras áreas del currículo y fomentando estrechas relaciones con los maestros de clase. Desde mi experiencia, y con la retroalimentación de mis estudiantes de Magisterio en prácticas, en la mayoría de los casos esto no se aplica, y, así, «profesor» de música puede tener un estatus más bajo (de media jornada o sin cualificación) y, por ello, verse aislado del resto de la escuela al no tener expectativas de trabajo ni tener la posibilidad de trabajar en estrecha relación con el resto del personal docente.

Esto puede tener tres resultados preocupantes: refuerza la noción de que la música es diferente y demasiado especializada para la mayor parte de los docentes (y quizás también para la mayoría del alumnado); se aparta de los procesos de planificación y desarrollo que comparte el conjunto del personal de la escuela; y, de forma más crucial, desde la perspectiva de la formación del profesorado, reduce las oportunidades de formación que tienen los docentes para aprender a enseñar música en su etapa de prácticas docentes.

Esto constituye una paradoja pues, como formadores de docentes, se supone que nuestra tarea consiste en preparar a los estudiantes para que sean profesores y profesoras capaces de enseñar el programa en su totalidad. En su centro de formación reciben una aportación bastante mínima -aunque habitualmente de gran calidad- y la mayoría de ellos se muestran entusiastas e impacientes por enseñar a los niños. Llegan a clase y se encuentran con que el maestro no enseña música en el aula y no espera tampoco que su «estudiante en prácticas» demuestre interés por enseñar música. La persona que enseña música es reacia a ser observada y puede no tener la experiencia de apoyar una práctica estudiantil. De este modo, muchos estudiantes denuncian la falta de oportunidades o al menos una limitación en las ocasiones que tienen de poder enseñar música y pierden rápidamente la confianza (Hennessy, 2010). Una minoría, por lo general los más cualificados y con mayor seguridad en sí mismos, puede gestionar estas oportunidades, pero es una desgracia que las políticas contradictorias hayan logrado traer consigo esta falta de coherencia.

Las políticas actuales para la formación de docentes se orientan cada vez más hacia una formación desarrollada en la escuela ${ }^{10}$ y esto podría tener graves consecuencias para el acceso a una buena práctica, o incluso a ninguna, de los futuros docentes de Educación Primaria. Actualmente, trabajamos asesorando y dando recursos a los formadores de docentes allá donde se encuentren (escuelas o centros de formación) para mejorar esta situación. El gobierno nos apoya en esta tarea $y$, aunque no esté íntegramente financiada, el nuevo espíritu de la comunidad profesional debería participar activamente tomando cartas en el asunto. Es demasiado pronto para evaluar el impacto que tiene este trabajo.

\section{Clase instrumental y clase vocal en grupo}

El segundo ejemplo es -de alguna manera- más profundo, ya que ha dado lugar a un cambio en la naturaleza de la música en tanto que asignatura en las escuelas.
Debido a una campaña de apoyo coordinada y muy lograda, encabezada por la comunidad educativa musical durante el último gobierno (Departamento de Educación y Competencias, 2004), la financiación estuvo garantizada para instaurar dos programas nacionales con el fin de apoyar la música en las escuelas Primarias. Uno se centró en el canto (http://www.Singup.org.uk), mientras que el otro lo hizo proporcionando a todos los niños el equivalente de un año gratuito de clases en grupo de un instrumento (Beach y otros, 2010). Esto fue un intento bien intencionado (aunque no del todo bien planificado) para tratar de compensar las pérdidas de la década anterior durante la cual la clase gratuita de instrumento había desaparecido virtualmente de las escuelas públicas debido a los cambios en el modo de financiación de las escuelas. El proyecto implicaba echar mano de profesorado instrumentista experto encargado de impartir clases en grupo, generalmente a lo largo de 3 trimestres. La elección del instrumento variaba según las tomas de decisiones locales. De este modo, los niños podían aprender a tocar el violín, la guitarra o el tamtan durante 10 semanas o más, algunas veces pasando por un instrumento diferente después de algunas semanas de aprendizaje. El concepto implica que los niños y niñas aprendan música mediante la experimentación instrumental antes que centrándose principalmente en las destrezas técnicas inherentes al aprendizaje de un instrumento (un cambio significativo en el modo de pensar de muchos profesores instrumentistas). La pericia para llevarlo a cabo correctamente ha evolucionado y puede ser excelente; se recomienda la implicación de los profesores de clase (aprendiendo al lado del estudiante y coenseñando con éste), aunque sea difícil hacerlo debido, en gran medida, a las cuestiones planteadas por la PPA, debido a que muchas escuelas programarán estas sesiones en el tiempo previsto para la PPA, y a que el profesorado instrumentista acepta en diferente modo esta nueva pedagogía colaborativa que no tiende únicamente hacia una técnica de enseñanza ni se concentra en el más capacitado. La iniciativa continúa con un descenso sustancial en cuanto a financiación se refiere. Abundan las interpretaciones individuales sobre: qué es lo mejor que puede incorporarse a este currículo; una falta de atención sobre cómo el profesorado de aula debería abordar este programa; y la ausencia continua de políticas de formación del profesorado instrumentista ${ }^{11}$, lo que lleva a una extensa fluctuación en la calidad de la enseñanza y en el nivel de resultados. El objetivo final que persigue esta iniciativa consiste en estimular el interés por aprender un instrumento más allá del primer año de gratuidad. Los datos no sugieren que el aprendizaje de la enseñanza instrumental más allá de ese primer año haya conocido un aumento significativo. (Bamford y Glinkowski, 2010).

Lo que es evidente ahora es el cambio en el énfasis que se ha puesto, tanto en la política relativa como en el currículo y en la práctica. Mientras que el Currículo Nacional promueve la interpretación, la composición y la audición, es evidente que el aprendizaje de las destrezas para ser un músico que sea buen intérprete predomina ahora en muchas escuelas. Mediante esta iniciativa de clase instrumental y de canto, muchas escuelas primarias esperan que sean los «expertos» los que enseñen música, y, que los profesores de asignaturas generales puedan (deban) renunciar sin consecuencias para ellos a sus responsabilidades de enseñar 
música en su aula. Las recientes declaraciones del gobierno al respecto consideran que la enseñanza del canto y el aprendizaje de un instrumento son una prioridad esencial (DfE, 2011, p. 7):

Sea cual sea su procedencia social así como su procedencia geográfica, en Inglaterra, los niños deberían tener la oportunidad de aprender un instrumento de música; hacer música con los demás; aprender a manejarse; y tener la oportunidad de progresar hasta el siguiente nivel de excelencia si así lo desean.

Un poco más adelante en el texto, se lee lo siguiente: ¿Qué experimentarán los niños? Programas de enseñanza para que todos los alumnos de una clase formen un 'ensemble' durante un año en el mejor de los casos (pero, como mínimo un trimestre); oportunidades para tocar en agrupaciones y para interpretar; una progresión clara, aprovechable y asequible; y una estrategia de canto que garantice que cada niño canta con regularidad (ibíd.).

Observaciones y conversaciones con profesores de música en la escuela aconsejan además que esta actividad se centre en mayor grado en niños interpretando en agrupaciones instrumentales y en coros antes que en un grupo creativo haciendo música, hecho que, sin embargo, juega un papel importante en las clases de música de Educación Primaria. Esto me lleva de nuevo a la cuestión de la formación docente. En este contexto, ¿quién está mejor situado para enseñar este tipo de currículo musical? Si las destrezas de ejecución, así como la experiencia, son ahora el foco principal, entonces existen pocas dudas sobre el hecho de que necesitamos, desde una etapa temprana, un profesorado de música especialista que pueda instruir y formar a estudiantes hasta lograr altos niveles de competencia musical. Con una educación musical «real» que se desarrolle, bien sea en una escuela privada o bien en escuelas de música especializadas, el profesor de enseñanza general pasará a estar considerado como una pobre sustitución y es probable que a la música en la escuela se la considere como bastante irrelevante.

\section{Formación musical del docente}

El actual sistema educativo exige unas mediciones rigurosas del rendimiento de las escuelas y del alumnado; se ve con recelo la evaluación realizada mediante control continuo y los exámenes presenciales «por escrito» están en auge; antes que confiar en el criterio profesional de los profesores ahora se exige un aprendizaje de la lectura musical. Al mismo tiempo (y para mayor confusión), las escuelas consideradas excelentes (por la inspección) tienen la libertad de desviarse del Currículo Nacional, de pagar a los profesores menos o más de lo estipulado en los convenios a nivel nacional y de contratar a profesorado no cualificado para asignaturas como la música o la educación física en las que se considera que la capacidad de rendimiento es el indicador predominante para la capacidad de enseñanza. Es decir, existe una especie de sector emergente independiente financiado por el Estado. La recompensa por un alto rendimiento se plasma en un cierto grado de autonomía, aunque dicha autonomía siga inmersa en el contexto de la inspección y de un sistema examinador con altas exigencias que limita el plan de estudios (Alexander y otros, 2009).

En el contenido del programa de música y en el contexto, más amplio, de la educación escolar tales cambios hacen incompatibles la formación del docente y la práctica profesional: el profesor tutor enseña cada vez menos música, mientras los programas de formación deben reflejar el papel tradicional ejercido por el tutor y formar a los estudiantes para que puedan enseñar el programa de forma íntegra (existen muy pocos cursos de formación especializada para maestros de Primaria en el Reino Unido). Las políticas actuales para la formación de los docentes tienden, cada vez más, a llevar la formación a las escuelas, lo que podría tener graves consecuencias para el acceso a una buena práctica o, incluso, a ninguna, de los futuros docentes de Educación Primaria. Ahora las escuelas son capaces también de formar a los propios docentes con un compromiso mínimo por parte de las instituciones universitarias y, en los cursos universitarios, los futuros docentes de Educación Primaria deben pasar una parte importante de su tiempo de formación en la escuela (más de la mitad en un año de programa).

Algunos nuevos desarrollos ofrecen la posibilidad de mejora, aunque nos movemos en terrenos movedizos a la hora de apoyar las declaraciones de intenciones con una acción financiada. The National Review of Music Education (DfE, 2011) fue un encargo del gobierno actual y sus recomendaciones se reflejan en el Plan Nacional para la Música (DfE, 2011) que incorporó al plan la oferta de un «módulo adicional» en la formación musical para los estudiantes de magisterio que había de llevarse a cabo hacia el final de su formación o una vez concluida. Aunque sus intenciones sean encomiables, no se entendió la imposibilidad de que esto funcione sin una financiación regular (las instituciones y/o los estudiantes tendrían que financiarlo). Afortunadamente prevaleció el sentido común y ahora nos hemos lanzado al desarrollo de un asesoramiento y recursos en línea para dar apoyo a los formadores en sus cursos de formación destinados a los docentes de música (bien en la escuela, bien en la universidad).

\section{Una perspectiva internacional}

Un interesante hallazgo del proyecto meNet consistió en descubrir las diferencias en los niveles de control gubernamental del sistema educativo en diferentes países de Europa. En los inicios de nuestro trabajo de desarrollo de los resultados de aprendizaje para la formación de los docentes de música (2007), empezamos a investigar cómo la formación docente del profesorado de música estaba estructurada y organizada en un centro de formación específico para cada uno de los siete países representados, expresando a muy grandes rasgos el rango geográfico y económico de los países implicados en el proyecto en su conjunto (Inglaterra, Portugal, España, Suecia, Grecia, Eslovenia y Países Bajos. Estas eran las instituciones en las que trabajaban los socios del proyecto). Entre el gran número de preguntas realizadas, hubo algunas que hacían referencia al grado de autonomía que tenían las instituciones en varios aspectos de su trabajo (contratación, plan de estudios, estructura del curso y evaluación). En este aspecto, encontramos que Inglaterra y Portugal disponían del grado más alto de control, muy centralizado, mientras que Grecia tenía el más bajo. Solo Inglaterra y Portugal trabajaban, en materia de contratación y hasta en la formación, según cuotas anuales impuestas por el gobierno. En España y Grecia, las instituciones no estaban autorizadas a hacer una selección para la música y debían aceptar a los estudiantes en base a exámenes generales de ingreso, sin entrevista. En Suecia, España e Inglaterra, el gobierno impone el tiempo que debe dedicarse a las prácticas docentes en la escuela. Todos los gobiernos prevén directrices generales así como una legislación relativa a la acreditación y 
a los requisitos de entrada. En Inglaterra, cada aspecto del proceso de formación está estrechamente controlado - desde los requisitos de entrada hasta los resultados (competencias docentes) de la formación-; no obstante, se deja un espacio para cierta flexibilidad en el diseño del curso. Mientras el control de la cuota de entrada de estudiantes sirva para evitar una superabundancia en la oferta, el establecimiento de unos requisitos mínimos para cada aspecto de la formación hace que sea difícil innovar, y, por otro lado, el establecimiento de cuotas puede configurar la profesión de forma radical (en los dos últimos años el número de contratación de formadores de docentes de música se ha visto reducido a la mitad).

La combinación de una estrecha supervisión (con consecuencias punitivas para los bajos rendimientos) con las restricciones presupuestarias con las que cuentan las universidades convierte a la formación del profesorado de Educación Primaria en una actividad especialmente complicada en las enseñanzas universitarias. El gobierno actual de Inglaterra tiene interés por transferir en mayor grado la formación de los docentes a las escuelas y ya ha empezado a reducir el papel de las universidades mientras, al mismo tiempo, quiere atraer a profesores de alto nivel (con el fin de emular a las estrellas de PISA: Finlandia y Singapur, olvidándose de manera muy oportuna de que ¡la formación de profesores en Finlandia conlleva 5 años de estudios universitarios!). Unas exigencias mínimas permiten una gran libertad a la hora de desmarcarse y de ser innovador pero, como se ha visto en Grecia, un resultado de tal libertad significa que hay centenares de profesores cualificados aunque sin empleo (incluso antes de la actual crisis) y el hecho de la falta de un proceso de selección controlado por las universidades puede conducir a elevados índices de abandono. El comentario de los que participaron en el estudio resaltó que la música en el contexto del plan de estudios en su totalidad corría el peligro de volverse cada vez más marginalizada (como ha sucedido en Inglaterra y en España). De igual manera, allá donde apenas haya reglamentación alguna, la música podrá tener muy poco asidero en los programas de formación, y reflejará y perpetuará de nuevo el bajo estatus del que goza en el currículo escolar.

Allá donde la formación del profesorado de música involucra a instituciones de música especializadas (como sucede en Suecia y en los Países Bajos), parece que existe más reconocimiento y respeto por parte del gobierno cara a la autonomía profesional de los formadores de música en ambos campos, bien sea en el de la formación artística, bien en el de la formación de los docentes. Inglaterra puede considerarse una de las pocas excepciones en Europa que da formación a todos los docentes en las facultades de educación de las universidades; fuera de estas facultades, tan solo existen un puñado de cursos que impliquen a los departamentos de música o a los conservatorios. Esto tiene por efecto aislar a los formadores de música (tan solo son, con frecuencia, uno o dos miembros del personal), lo que dificulta, en ocasiones, la posibilidad de cubrir la extensa variedad de conocimientos y destrezas exigidas por el plan de estudios en las escuelas.

La situación que afecta a la formación en la Educación Primaria es diferente. Dado que no hay apenas formación especializada para la música en este nivel educativo para el profesorado especialista, la presencia de la música en los programas de formación depende de si la institución se toma el tema con la suficiente seriedad como para crear un puesto académico a tal efecto o se limita a contratar a un maestro de Primaria sobre la base de una retribución por horas para un curso de corta duración. Algo parecido ocurre en las escuelas en donde la contratación de un profesor de música experto depende de la decisión del director del centro. De este modo, son los intereses y entusiasmos de una persona los que gobiernan la calidad de la educación musical para el alumnado más joven así como para los maestros que les enseñan la materia.

\section{Apoyo}

Lo que acabo de presentar aquí da una visión parcial de la situación en Inglaterra, a pesar de lo cual refleja, en gran parte, una imagen bastante incoherente. Esto sirve para ilustrar que lo que puede tardar muchos años en desarrollarse puede perderse muy rápidamente mediante decisiones políticas que tienen muy poco en cuenta el conocimiento profesional y la experiencia. El apoyo en defensa de la asignatura es, sin duda, crucial y es una necesidad siempre presente: la realización de campañas llevadas a cabo por los formadores de música, con frecuencia con el apoyo de músicos profesionales, puede lograr influenciar o incluso imprimir un cambio de política. Sin embargo, tales campañas necesitan unos objetivos claramente definidos, pues pueden desviar la atención sobre lo que considero que son los aspectos más importantes en la educación musical. Es decir, lo que brindamos a todos los jóvenes sin reparar en su lugar de residencia o su situación económica o social y, en esta disposición, se trata de ofrecerles la mejor enseñanza posible llevada a cabo por un profesorado correctamente formado y apoyado. Estas campañas en favor de la educación musical escolar pueden ser impactantes y eficientes, pero a menudo su mensaje tiene que ser simplificado para poder servir en el contexto político. También se da el caso, con frecuencia, de que la atención y la financiación obtenidas gracias a estas campañas se ven limitadas en el tiempo, lo que dificulta el desarrollo de proyectos coherentes y bien planificados que lleven a unos cambios duraderos y a unas mejoras en la práctica. Un ejemplo de ello es la aprobación de proyectos como «El Sistema», financiados por el gobierno y por músicos como Julian Lloyd Webber. La expectación y el glamour suscitados por «El Sistema» han hecho que los gobiernos de muchos países se hayan desvivido por poner en marcha, para lo ya existente a nivel local -y con un entusiasmo casi apostólico que tiende a excluir la evaluación crítica-, proyectos, que en algunos casos son de poca envergadura:

'El Sistema Escocia' ha adoptado una perspectiva profesional que da prioridad a una necesidad con una fe incondicional en el valor intrínseco y en la eficiencia del programa. Esto [...] no sucede sin limitaciones. Podría verse como para incorporar [...] una postura "apostólica" en relación con la [...] comunidad dependiendo de las declaraciones de fe en el proceso de todos los individuos involucrados en ella (Allan y otros, 2010, p. 344).

La evaluación de Bamford (2011) sobre la política denominada «Cultural Rucksack» (DKS) en Noruega subraya otro asunto preocupante como es la falta de atención prestada al desarrollo profesional de los docentes y artistas para desarrollar y mantener su compromiso con el aprendizaje de los escolares:

Esta política facilita una financiación generosa de las escuelas para permitir a los niños entrar en contacto con 
artistas profesionales de todos los estilos; sin embargo, el actual DKS parece tan solo tener un impacto fugaz en los estudiantes. Esto puede ser porque los conciertos vienen y van sin formar parte de un programa continuo, o bien porque el alumnado no percibe la importancia de esos conciertos en su propio interés o para su experiencia (p. 10).

La experiencia inglesa demuestra cómo las campañas de apoyo pueden producir -en el momento oportuno- unos resultados impresionantes: Son 10 millones de libras los que se destinaron a las iniciativas musicales Sing Up y WCVIT Initiatives durantes 3 años, y aproximadamente 5 millones de libras los que se dedicaron al programa denominado In Harmony (El Sistema inspiró ocho proyectos en zonas necesitadas) pero, al mismo tiempo, la simple tarea cotidiana y esencial de brindar a todos los niños una educación musical «suficientemente buena» no cuenta, lamentablemente, con suficientes recursos y está mal comprendida por parte de los responsables políticos.

En cuanto a nuestras aptitudes como formadores de docentes, la responsabilidad de quienes nos dedicamos a la de música debería ser la de preparar a los nuevos docentes para ser críticos, para articular y para ser conscientes del contexto político en el que se encuentran y en el que tendrán que ejercer la enseñanza; desarrollar redes a través de las cuales los docentes puedan aprender unos de otros; recibir consejos, apoyar y encontrar la propia voz profesional para desafiar e influenciar a las políticas. Como investigadores, necesitamos generar datos de investigación fiables, no solo para apoyar, sino también para cambiar y mejorar la calidad del aprendizaje musical al que todo niño tiene derecho.

\section{Notas}

${ }^{1}$ Periódico The Guardian, 12/07/12. http://

www.theguardian.com/uk/feedarticle/10333068.

2 "Major international study shows England's 15-year-olds performing poorly in mathematics, science and reading". Comunidado de prensa del Departamento de Educación británico (2010). Disponible en http://

www.education.gov.uk/inthenews/inthenews/a0070008/ secretary-of-state-comments-on-pisa-study-of-school-systems. ${ }^{3}$ Periódico The Telegraph, 23/08/13. http://

www.telegraph.co.uk/education/educationnews/10258795/ GCSE-results-2013-record-fall-in-top-grades-as-tough-examreforms-take-effect.html.

${ }^{4}$ Correo electrónico personal del Dr C. Rolle, enero de 2013. ${ }^{5}$ Es importante saber que existen cuatro sistemas educativos distintos en el Reino Unido, cada uno de ellos tiene su propio enfoque sobre el plan de estudios, la evaluación, la inspección y la formación del profesorado, aunque se puede observar un grado de similitud entre ellos (para la mayor parte del siglo pasado, Westminster era el responsable directo de las políticas educativas así como de su financiación en Gales, Irlanda del Norte e Inglaterra).

${ }^{6}$ meNet fue un proyecto Comenius 3 (2006-2009) financiado por la UE centrado en el intercambio del conocimiento en el campo de la educación musical en Europa. Dos de las tareas a realizar consistieron en recopilar información con comentarios sobre la educación musical en la escuela y la formación del profesorado en 20 países europeos. La tercera tarea consistió en crear resultados de aprendizaje para la formación del profesorado de música. Los resultados pueden verse en http://www.menet.info.
${ }^{7}$ Véase, por ejemplo, http://www.meNet.info; Russell Bowie, 2009).

${ }^{8}$ No se dispone de datos específicos al respecto - por lo que he acudido a mi conocimiento de las escuelas a las que nuestros estudiantes de formación del profesorado fueron destinados, así como a los datos empíricos basados en la comunidad de la educación musical y a los anuncios de puestos de trabajo fijo para el profesorado de música.

${ }^{9} \mathrm{El}$ gobierno aconseja ahora a las escuelas que contraten a profesorado sin cualificación que sean «músicos expertos» ante la creencia simplista según la cual un músico experto es inevitablemente más idóneo para enseñar que un profesor, iy más económico, por supuesto!

${ }^{10}$ School Direct es una reciente iniciativa promovida por el gobierno actual. Desde 2013, las escuelas pueden dedicarse a contratar a estudiantes en prácticas, de modo que los estudiantes son acogidos por las escuelas en vez de por un centro de formación. Las escuelas eligen con quién trabajar en colaboración y acuerdan los costes, de modo que el proceso de formación pasa de la universidad a las escuelas. ${ }^{11}$ No se exige que los profesores instrumentistas dispongan de cualificación para la enseñanza.

\section{Referencias Citadas}

Alexander, R. J. (2009). Research, the Cambridge Primary Review and the quality of education. En R. J. Alexander, C. Doddington, J. Gray, L. Hargreaves, y R.Kershner (Ed.) (2009). The Cambridge Primary Review Research Surveys. Londres: Routledge.

Alexander, R. (2012). International evidence; national policy and classroom practice: Questions of judgement vision and trust. Ponencia presentada dentro de $3^{\text {er }}$ Congreso Internacional Van Leer sobre educación. Jerusalén, 24 de mayo. Disponible en http://cprtrust.org.uk/wp-content/ uploads/2014/06/20120524_Van_Leer_Alexander.pdf.

Allan, J., Moran, N., Duffy, C., y Loening, G. (2010). Knowledge exchange with Sistema Scotland. Fournal of Educational Policy, 25(3), 335-347. DOI: 10.1080/02680931003646196

Ball, S. (1998). Big policies/small world: An introduction to international perspectives in education policy. Comparative Education, 34(2), 119-130.

Baird J-A, Isaacs, T., Johnson, S., Stobart, S, Yu, G., Sprague, T., y Daugherty, R. (2011). Policy effects of PISA. Oxford University Centre for Educational Assessment. Disponible en http://oucea.education.ox.ac.uk/wordpress/wpcontent/uploads/2011/10/Policy-Effects-of-PISAOUCEA.pdf.

Bamford, A. (2011). Arts and Cultural Education in Norway. Disponible en http://www.musikerorg.no/_upl/ arts_and_cultural_education_in_norwayl.pdf.

Bamford, A. y Glinkowski, P. (2010). Wow it's Music Next! The impact of wider opportunities. Disponible en http:// www.somersetmusic.somerset.org.uk/Documents/Tuition $\% 20$ Documents / Wow, $\% 20$ it $\% 27 \mathrm{~s} \% 20$ music $\% 20$ next.pdf.

Beach, N., Evans, J. y Spruce, G. (2010). Making music in the primary school: Whole class instrumental and vocal teaching. Londres: Routledge.

Carter, D. S. G. y O'Neill, M. H. (1995). International Perspectives on Educational Reform and Policy Implementation. Brighton: Falmer. 
Chawla-Duggan, R. y Lowe, J. (2008). Aims for primary education: changing global contexts; Primary Review Research briefings 1/4. Disponible en http:// cprtrust.org.uk/wp-content/uploads/2013/10/

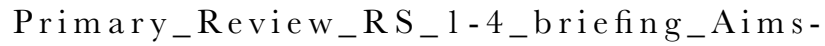
global_contexts_080118.pdf.

Coffield, F. (2012). Why the McKinsey reports will not improve school systems. Fournal of Education Policy, 27(1), 131-149. DOI: 10.1080/02680939.2011.623243

Department of Education (2011). The importance of music: $A$ national plan for music. Disponible en https://www.gov.uk/ government/publications/the-importance-of-music-anational-plan-for-music-education.

Department of Education (2010). Major international study shows England's 15-year-olds performing poorly in mathematics, science and reading. Disponible en http:// www.education.gov.uk/inthenews/inthenews/a0070008/ secretary-of-state-comments-on-pisa-study-of-schoolsystems.

Department for Education and Skills (2004). Music Manifesto. Disponible en http://webarchive.nationalarchives.gov.uk/ 20130401151715 /http:/www.education.gov.uk/ publications / standard/publicationdetail / page 1/1-84478-533-5.

EACEA (2009) Arts and cultural education at school in Europe. Bruselas: Education, Audiovisual and Culture Executive Agency. Disponible en http://eacea.ec.europa.eu/ education/eurydice/documents/thematic_reports/ 113EN.pdf.

GloverJ. y Ward S. (1998). Teaching music in the primary school. Londres: Continuum.

Greevy, H., Knox, A. Nunney, F., y Pye, J. (2012). The effects of the English Baccalaureate. Department for Education. Disponible en http://dera.ioe.ac.uk/15641/1/DFERR249.pdf.

Hennessy S (2010). La formazione musicale degli insegnanti della scuola primaria. En M. Biasutti (Ed.). Educare a Educare. Pensa: MultiMedia Editore.

Hennessy, S., Rolfe, L., Chedzoy, S. (2001). The Factors which influence student teachers' confidence to teach the arts in the primary classroom. Research in Dance Education, 2(1), 53-71.

Holden, H. y Button, S. (2006). The teaching of music in the primary school by the non-music specialist. British fournal of Music Education. 23(1), 23-38.

Mills J. (1989). The generalist primary teacher of music: a problem of confidence. British Fournal of Music Education, 6(2), 125-138.

Paynter, J. (1970). Sound and Silence. Oxford: OUP.

Russell-Bowie, D. (2009). What me? Teach music to my primary class? Challenges to teaching music in primary schools in five countries. Music Education Research, 11(1), 23-36.

Shuayb M. \& O'Donnell, S. (2008) Aims and values in primary education: Primary Review Research Survey 1 / 2. Disponible en https://www.nfer.ac.uk/publications/ PRR01/PRR01.pdf.

Swanwick, K. (1988). Music mind and education. Londores: Routledge.

UNESCO (2006). World Map for Arts Education. Disponible en h t t p : / / w w w. un esco.org/new/fileadmin /
M U L T I M E D I A / H Q / G L T / C L T / p d f / Arts_Edu_RoadMap_en.pdf.

Woodford, P. (2005). Democracy and music education: Liberalism, ethics, and the politics of practice. Bloomington: Indiana University Press. 


\section{Sobre la Autora}

\section{Sarah Hennessey}

Sarah Hennessey es Profesora Titular de educación musical en la Universidad de Exeter (Reino Unido), en donde coordina el curso de postgrado de formación del profesorado (PGCE). También es coordinadora de los programas de máster y directora de tesis doctorales. Sus campos de trabajo son fundamentalmente los de formación del profesorado especialista y generalista; enfoques creativos en enseñanza y aprendizaje; y el trabajo de artistas profesionales en educación. Es editora fundadora de la revista Music Education Research y directora del congreso internacional de Investigación en Educación Musical (RIME) que se celebra cada dos años. Ha sido evaluadora de proyectos educativos de la Orquesta Sinfónica de Londres, la Orquesta del Siglo de las Luces, y la Real Casa de la Ópera de Londres, entre otros muchos. Actualmente está trabajando con la Agencia Estatal de Enseñanza (Teaching Agency) para desarrollar formación musical complementaria para el profesorado de Primaria. Ha sido presidenta de la Asociación Nacional para la Educación Multicultural del Reino Unido (NAME) y de la Asociación Europea para la Música Escolar (EAS) y actualmente es miembro electa de la Junta Directiva de la Sociedad Internacional para la Educación Musical (ISME).

\section{Sarah Hennessey}

Universidad de Exeter

Campus de St Lukes

Heavitree Road

Exeter EX1 2LU (Reino Unido)

S.J.E.Hennessy@exeter.ac.uk

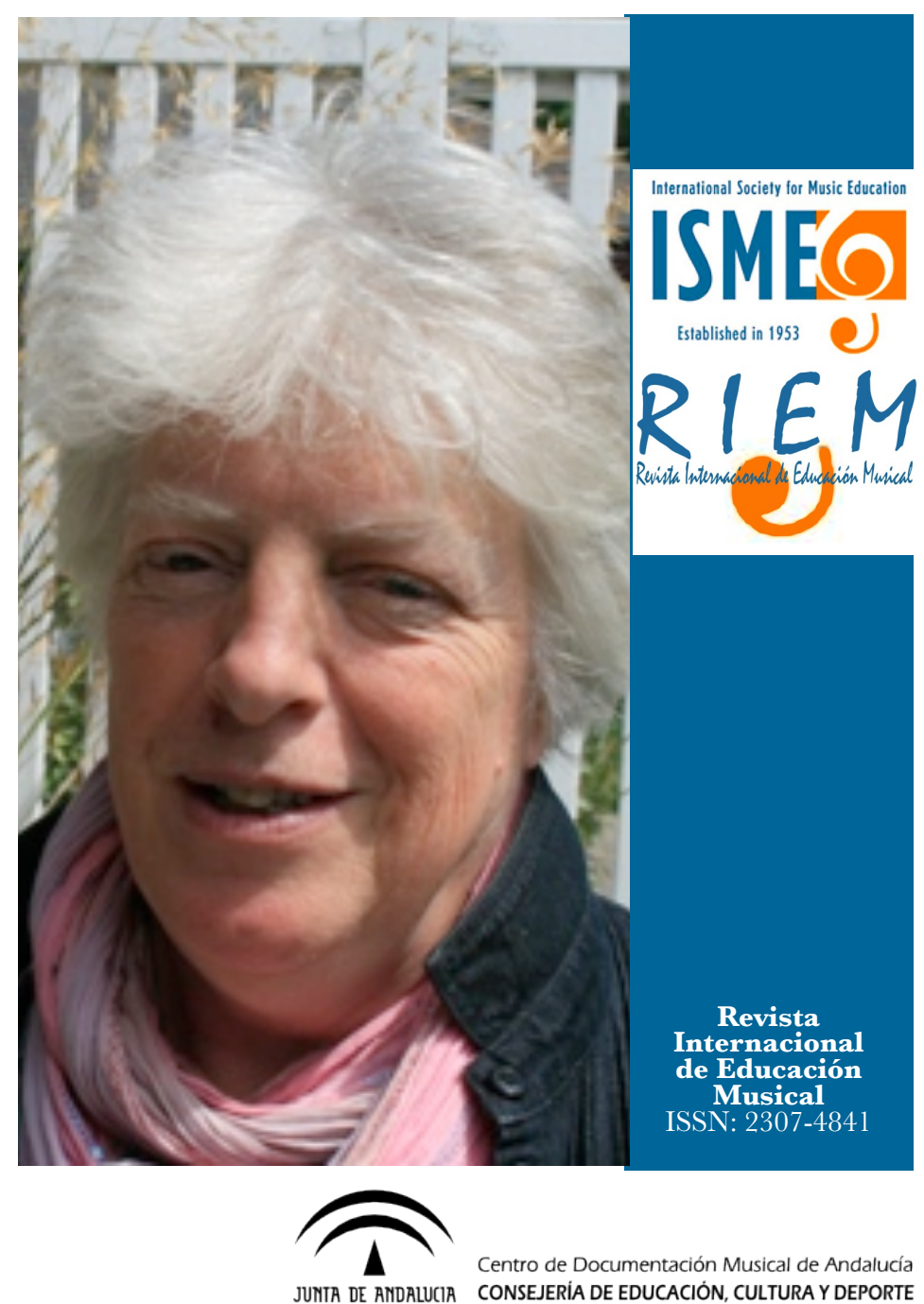

\section{EQUPO EDITORIAL}

Editor:

José Luis Aróstegui Plaza, Universidad de Granada (España)

\section{Editora Adjunta:}

Rosa María Serrano Pastor, Universidad de Zaragoza (España)

\section{Consejo Editorial}

Carlos Abril, Universidad de Miami (Estados Unidos)

María del Carmen Aguilar, Instituto Coral de Buenos Aires (Argentina)

Miquel Alsina Tarrés, Universidad de Gerona (España)

Carmen Carrillo Aguilera. Universidad Internacional de Cataluña (España)

Sergio Luiz Figueiredo, Universidad del Estado de Santa Catarina (Brasil)

Patricia Adelaida González, Universidad Autónoma de Chihuahua (México)

Claudia Gluschankof, Instituto Levinsky (Israel)

Josep Gustems Carnicer, Universidad de Barcelona (España)

María Cecilia Jorquera Jaramillo, Universidad de Sevilla (España)

Gotzon Ibarretxe Txakartegi, Universidad del País Vasco (España)

Ana Laucirica Larrinaga, Universidad Pública de Navarra (España)

Ana Lucia Louro, Universidad Federal de Santa María (Brasil)
Isabel Cecilia Martínez, Universidad Nacional de La Plata (Argentina)

Teresa Mateiro, Universidad del Estado de Santa Catarina (Brasil)

María Teresa Moreno, Universidad Laval (Canadá)

Graça Boal Palheiros, Instituto de Educación de Oporto (Portugal)

Jèssica Pérez Moreno, Universidad Autónoma de Barcelona (España) y Universidad de Londres (Reino Unido)

Gabriel Enrique Rusinek Milner, Universidad Complutense de Madrid (España)

Patrick K. Schmidt, Universidad de Ontario Occidental (Canadá)

Favio Shifres, Universidad Nacional de La Plata (Argentina)

Maria dels Àngels Subirats Bayego, Universidad de Barcelona (España)

António Ângelo Ferreira Vasconcelos, Instituto de Educación de Setúbal (Portugal)

Maria Helena Vieira, Universidad del Miño (Portugal)

Gloria Patricia Zapata Restrepo, Universidad de Antioquía (Colombia) 